\title{
Persistent Median Artery-An Uncommon Cause of Carpal Tunnel Syndrome
}

\author{
Reid A. Waldman ${ }^{+}$, Charles D. Donohoe* \\ Department of Neurology, University of Missouri-Kansas City School of Medicine \\ ${ }^{+}$MD candidate UMKC School of Medicine 2411 Holmes Kansas City MO. 64108, USA \\ *Professor and Interim Chair Department of Neurology UMKC School of Medicine 2411 Holmes Kansas \\ City MO. 64108, USA
}

\section{INTRODUCTION}

Carpal tunnel syndrome (CTS) is the most common peripheral entrapment neuropathy. It is caused by entrapment of the median nerve as it traverses the carpal tunnel beneath the transverse carpal ligament. Clinically, this entrapment neuropathy presents as nocturnal pain and hypesthesia in the distribution of the median nerve with associated weakness and possible atrophy of the corresponding muscles. While carpal tunnel syndrome is most commonly caused by tenosynovitis of the flexor tendons, anything that can compromise the median nerve within the carpal tunnel can serve as the nidus of the clinical syndrome, e.g. tumor, aneurysm, synovitis, acromegaly, thickening of the transverse carpal ligament to name a few (Fig. 1). Among these are a number of anatomic variants that have been increasingly described since the proliferation of the use of point of-care ultrasound in the diagnosis of CTS. The most commonly described anatomic variants are bifid median nerve and persistent median artery (PMA). ${ }^{1}$ It is important to identify these variants in patients suffering from carpal tunnel syndrome as their presence can alter both clinical outcome and management decisions. This article will review the ultrasound findings and clinical significant of CTS in the setting of persistent median artery with an emphasis on pathophysiology and management considerations.



Fig1. Thickened and bulging transverse carpal ligament in patient with carpal tunnel syndrome. The transverse ligament measures $0.8 \mathrm{~mm}$. Note the enlargement of the median nerve with loss of the normal internal architecture 


\section{EMBRYOLOGY OF PERSISTENT MEDIAN ARTERY}

A persistent median artery is an embryologic remnant that is present in up to one fifth of the population. ${ }^{1}$ The median artery forms early in embryologic development alongside the median nerve prior to the development of the radial and ulnar arteries and provides the majority of the initial blood flow to the hand as the embryo develops. While this artery usually involutes via apoptosis around 8 weeks of gestation, it occasionally persists within the carpal tunnel. Estimates of the frequency of persistence are highly varied ranging from $1.2 \%$ to $23 \%$ and are dependent on race, gender, and age. ${ }^{2}$ The majority of individuals with PMA, are completely asymptomatic. In fact, a recent study did not demonstrate any increased risk of developing CTS in patients with a PMA, although this study was woefully underpowered (Beta $<25 \%$ ). ${ }^{2}$ Additionally, it is worth noting that the presence of a persistent median artery has been associated with the presence of a bifid/trifid median nerve which has also been described as a cause of CTS.

Ultrasound has gained traction for the diagnosis of CTS as it is safe, effective, can facilitate rapid diagnosis and treatment via ultrasound guided nerve blocks as it can be performed by the treating physician at the point-of-care. The ultrasound diagnosis of carpal tunnel syndrome is straightforward and it accuracy compares favorably with nerve conduction velocity testing and electromyography. An unintended result of the increasing use of ultrasound in the diagnosis of carpal tunnel syndrome has been a concomitant increase in reports of anatomic variants causing CTS as this modality is able to characterize the local anatomy in great detail. Specifically, while persistent median artery has been reported as a cause of CTS for over 30 years, its significance has been increasingly highlighted as diagnostic ultrasound in the diagnosis of CTS has grown in popularity. The presence of a persistent median artery on ultrasound is described as tubular structure adjacent to the median nerve with unilateral flow on Doppler (Fig. 1). In cases of CTS resulting from persistent median artery, three variants have been described: enlarged artery, thrombosed artery, and median nerve intersection.

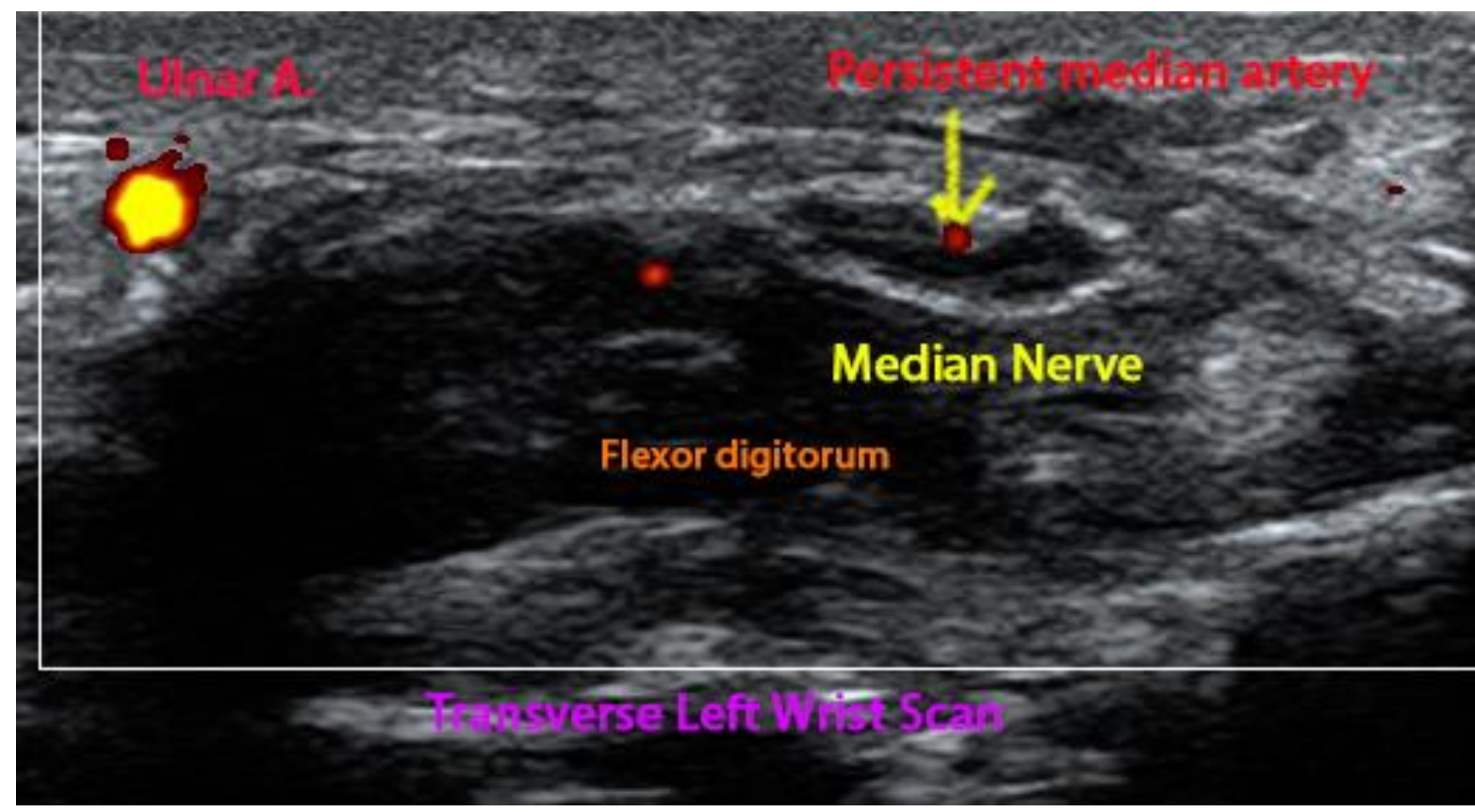

Fig2. Transverse color Doppler image in a woman with carpal tunnel syndrome demonstrating a patent persistent median artery (arrow).

When CTS occurs in the setting of an enlarged PMA ( $>1 \mathrm{~mm}$ lumen), it usually presents with subacute development similar to CTS secondary to tenosynovitis. ${ }^{3}$ Identifying an enlarged PMA is important as these patients will likely fail conservative therapy and nerve block and ultimately require carpal tunnel release for relief of symptoms. There are two major surgical considerations in these patients: 1) the PMA should not be ligated during surgery unless a detailed evaluation of the distal vasculature has revealed that it plays a non-integral part in blood flow as ligation is often not necessary for resolution of symptoms and can result in hand ischemia ${ }^{4}$; and, 2) patients with PMA are at an increased risk of carpal tunnel release failure and this risk is directly correlated with PMA lumen diameter (risk greatest when $>2 \mathrm{~mm})^{5}$ 
Reid A. Waldman \& Charles D. Donohoe "Persistent Median Artery-An Uncommon Cause of Carpal Tunnel Syndrome"

Contrasting the subacute course that patients with enlarged PMA follow, patients with thrombosed PMA may develop acute CTS symptoms. ${ }^{6}$ The development of a thrombosed PMA has been reported both spontaneously and after trauma and does not require the presence of a concomitant clotting disorder. These cases are unique in that patients can exhibit physical exam findings of a tender, warm, mobile nodule at the area of thrombosis. Identifying cases of PMA thrombosis is of utmost importance in that it directly impacts management as these patients have been successfully treated with 3 months of anticoagulation. ${ }^{7}$ In patients who fail anticoagulation, surgical removal of the thrombosed vasculature is curative.

Ultimately, ultrasound is an amazing diagnostic modality for CTS as it is not only effective and affordable, but also it provides detailed characterization of the anatomy allowing for identification of novel causes of CTS. Its utility is highlighted by cases where a persistent median artery is implicated as identification of PMA has serious clinical implications and can improve patient safety outcomes through tailored care and the avoidance of unnecessary intervention.

\section{REFERENCES}

[1] Gassner EM, Schocke M, Peer S, Schwabegger A, Jaschke W, Bodner G. Persistent Median Artery in the Carpal Tunnel: Color Doppler Ultrasonographic Findings. Journal of Ultrasound in Medicine. 2002;21:455.

[2] Altinkaya N, Leblebici B. Prevalence of persistent median artery in carpal tunnel syndrome: sonographic assessment. Surgical and Radiologic Anatomy. 2016;38:511-515.

[3] Lee Spangler M, Lee Spangler M, Almusa E, Britton C. Journal of musculoskeletal research: A Peristent Median Artery and Bifid Median Nerve in the Setting of Carpal Tunnel Syndrome. World Scientific Publishing Company; 06/01/2012;15:1272004.

[4] Bijannejad D, Azandeh S, Javadnia F, Gholami MR. Case reports in plastic surgery and hand surgery: Persistent median artery in the carpal tunnel and anastomosis with superficial palmar arch. Informa Healthcare; 2016;3:25.

[5] Derincek A, Adiyaman S, Demirtas AM, Bilgin SS. Archives of orthopaedic and trauma surgery: Can simple release relieve symptoms of carpal tunnel syndrome caused by a persistent median artery? Springer; 04/01/2004;124:154.

[6] Salter M, Sinha NR, Szmigielski W. Thrombosed persistent median artery causing carpal tunnel syndrome associated with bifurcated median nerve: A case report. Polish Journal of Radiology. 2011; 76(2):46-48.

[7] Rzepecka-Wejs L, Multan A, Konarzewska A. Journal of ultrasonography: Thrombosis of the persistent median artery as a cause of carpal tunnel syndrome - case study. Medical Communications Sp; 12/01/2012;12:487.

Citation: Reid A. Waldman, Charles D. Donohoe Persistent Median Artery-An Uncommon Cause of Carpal Tunnel Syndrome International Journal of Research Studies in Medical and Health Sciences. 2017; 2(1):1-3.

Copyright: (c) 2017 Reid A. Waldman, Charles D. Donohoe. This is an open-access article distributed under the terms of the Creative Commons Attribution License, which permits unrestricted use, distribution, and reproduction in any medium, provided the original author and source are credited. 